\title{
A method for determining the spatial attitude of the mooring system
}

\author{
Shan Gao ${ }^{1, \text { a) }}$ \\ ${ }^{1}$ North China Electric Power University, Baoding 071000,China. \\ a)1786831655@qq.com
}

\begin{abstract}
In this paper, the stress condition and design method of single point mooring system in shallow water observation network are studied.We analyze the force condition of the whole system,through the stress point of the lower end of the chain hypothesis,we can get the relationship between the depth of water and the stress of each part of the system,and find out the spatial distribution of each part.According to different situations, we can use this method to design the mooring system.In this paper, the problem of different wind speed and water flow is also discussed,and the corresponding solutions are given.
\end{abstract}

Keywords: Mooring System, Moment-equilibrium, Force analysis, binary chop

\section{INTRODUCTION}

The transmission nodes of near shallow sea observation network are composed of buoy system, mooring system and underwater acoustic communication system. The buoy system is simplified to the shape of cylinder, the mooring system is composed of a steel pipe (4 section), steel drums, heavy ball, welding anchor and special anti drag anchor.The angle between the tangent direction of the link chain requirements and the end of the anchor and seabed does not exceed 16 degrees,Or the anchor will be towed away.The underwater acoustic communication system is installed in a sealed cylinder.When the vertical steel drum, underwater acoustic communication equipment work best. If the drum tilt, will influence the equipment working effect.When the tilt angle (the angle between the steel drums and vertical lines) more than 5 degrees, the poor equipment working effect.We can hang heavy ball to control the inclination angle steel.We mainly study the attitude of each part of the mooring system.

Known parameters are as follows:

Buoy cylinder: diameter 2m, high 2m, mass $1000 \mathrm{~kg}$

Anchor: 600kg

Steel pipe:length $1 \mathrm{~m}$, diameter $50 \mathrm{~mm}$, mass $10 \mathrm{~kg}$

Sealed cylinder:length $1 \mathrm{~m}$, diameter $30 \mathrm{~cm}$, total mass $100 \mathrm{~kg}$

Anchor: length $105 \mathrm{~mm}$ (a section), mass per unit length $7 \mathrm{~kg} / \mathrm{m}$

Schematic diagram is as follows:

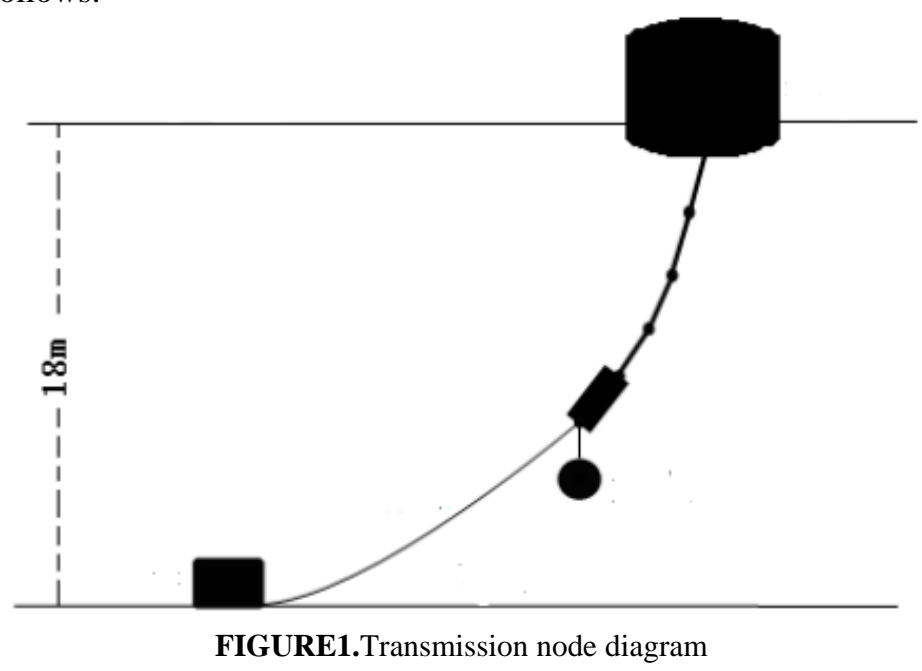

\section{ASSUMPTIONS}

1.It is assumed that all elements in the system are rigid bodies, which can not be elastically deformed.

2.Assuming that the flow velocity is uniform, ignoring the wave action.

3.Center of gravity hypothesis and equipment at the geometric center of steel drums.

4.Assuming that the heavy ball is a solid steel ball. 
5.Offshore wind load can be calculated by the approximate formula $F=0.625 \times \operatorname{Sv} 2(\mathrm{~N}), \mathrm{S}$ is the projection of wind in normal plane. $v$ is wind's velocity.

6.Near sea water flow force can be calculated by approximate formula $F=374 \times \operatorname{Sv} 2(N)$.S is the projection of flow in normal plane. $v$ is flow velocity .

\section{SYMBOL SPECIFICATION}

TABLE1.Symbol and its meaning

\begin{tabular}{|c|c|}
\hline symbol & meaning \\
\hline$P$ & the sum of the buoyancy of the system $(\mathrm{N})$ \\
\hline$G$ & the sum of the gravity of the system $(\mathrm{N})$ \\
\hline$P_{i}$ & buoyancy of parts themselves $(\mathrm{N})$ \\
\hline$G_{i}$ & gravity of parts themselves $(\mathrm{N})$ \\
\hline$\theta_{i}$ & the angle between the pull force and the horizontal direction of the parts $\left(\left|\theta_{i}\right| \leq 90^{\circ}\right)$ \\
\hline$F$ & wind power(N) \\
\hline$\alpha_{i}$ & angle of part and horizontal direction \\
\hline$T_{\mathrm{i}}$ & tension between parts $(\mathrm{N})$ \\
\hline$M_{T}$ & moment(tension)(N) \\
\hline$M_{G P}$ & moment(Gravity and buoyancy)(N) \\
\hline$m_{0}$ & mass of heavy ball (kg) \\
\hline$F_{s}$ & static friction of anchor(N) \\
\hline$F_{N}$ & support of seabed to anchor(N) \\
\hline$\rho$ & seawater density $(\mathrm{kg} / \mathrm{m})$ \\
\hline$m$ & mass of buoy(kg) \\
\hline$S^{\prime}$ & base of buoy $\left(\mathrm{m}^{2}\right)$ \\
\hline$H$ & height of buoy(m) \\
\hline$l$ & bottom diameter of buoy(m) \\
\hline
\end{tabular}

\section{ESTABLISHMENT OF SPATIAL MODEL \\ Balance of forces}

In this paper, the spatial coordinate system is established based on the anchor point.We take the direction of water flow and wind force for $\mathrm{X}$ axis forward,take vertical direction as $\mathrm{Z}$ axis. ${ }^{[1]} \mathrm{We}$ have made the stress diagram of system as follows:

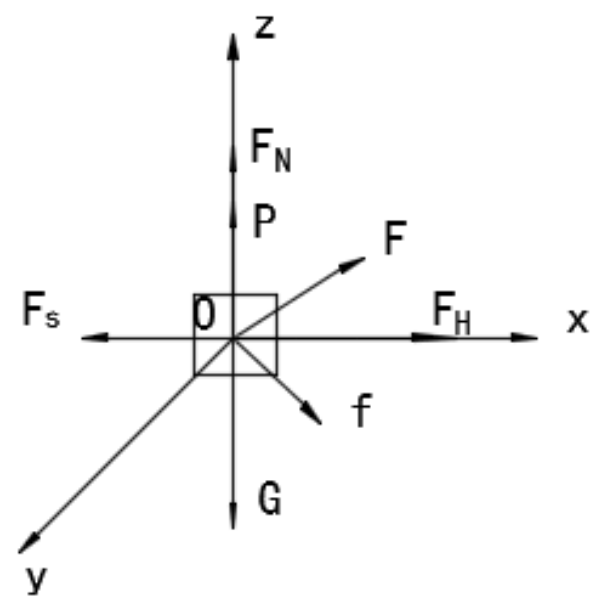

FIGURE2.stress diagram of system 
Because of the force balance of the system,we know $\vec{F}_{H}=\vec{f}+\vec{F}=-\vec{F}_{S}$, so our analysis of the anchor is as follows:

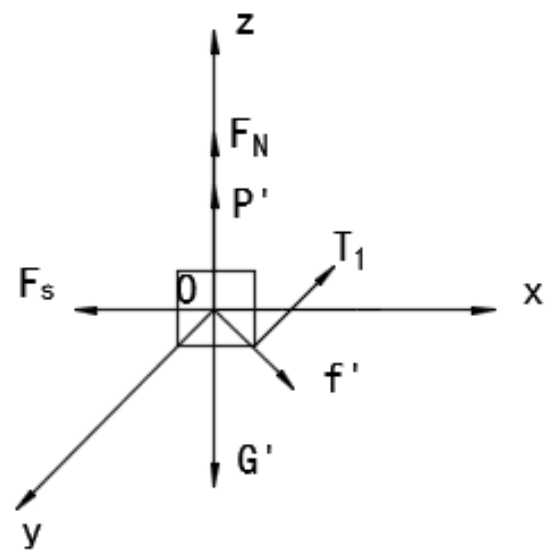

FIGURE3.stress diagram of anchor

Anchor received force of friction $\left(F_{S}\right)$,force of wind and flow $\left(F_{H}\right)$,stamina of seabed( $\left.F_{N}\right)$, tension of anchor chain $\left(T_{1}\right.$ ),gravity and buoyancy $\left(G^{\prime}\right.$ and $\left.P^{\prime}\right)$.Because of the force balance of the anchor,is the force on plane $X o Z$,and $F_{H}$ is the component in the $\mathrm{x}$ direction. $F_{H} \tan \theta_{1}$ is the component in the $\mathrm{z}$ direction.

Than,we analysis the stress condition of the first anchor chain,we can show all of the forces through the following figure.

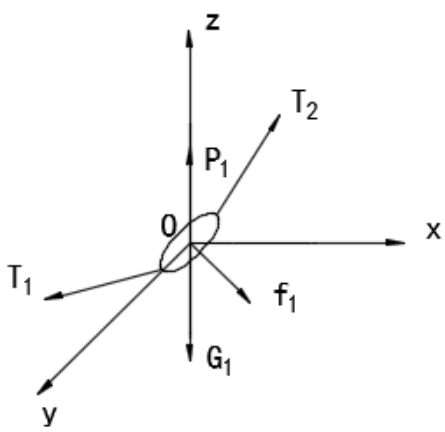

FIGURE4.stress diagram of the first anchor chain

Its force include:gravity and buoyancy,tension between anchor and chain $\left(T_{1}\right)$,the angle between the force and the plane is $\theta_{1}$,the force of the next section chain is $T_{2}$, the angle is $\theta_{2}$, Through the analysis of its force,we can get components in every direction.By this way,we can know the tension of the last chain $\left(T_{i+1}\right)$,its component in $\mathrm{x}$ direction is $F_{H}+\sum_{m=1}^{i} f_{m x}, \sum_{m=1}^{i} f_{m y}$ in y direction, $F_{H} \tan \theta_{1}+\sum_{m=1}^{i}\left(G_{m}-P_{m}\right)$ in z direction.

Due to the influence of the metal ball,we analysis the stress condition of steel pipes and the steel drum(we take the steel drum as example).First,we analysis the stress condition of the heavy ball.

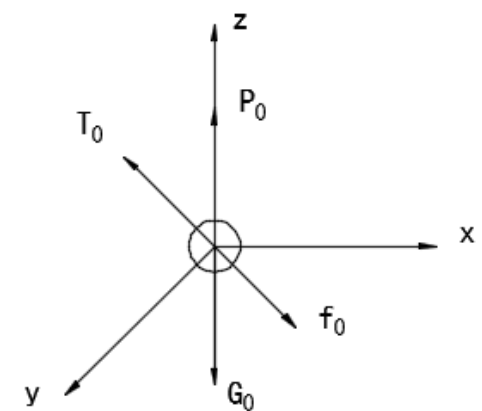

FIGURE5.stress diagram of the heavy ball

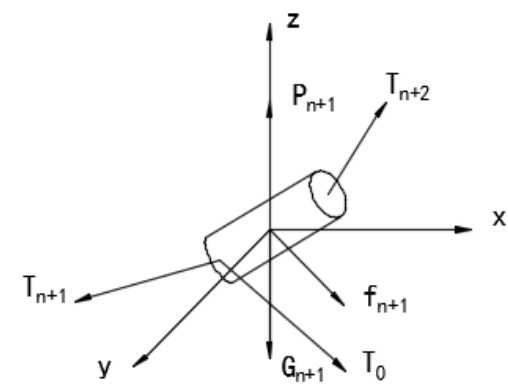

FIGURE6.stress diagram of the steel drum 
The component of $T_{0}$ in x direction is $f_{0 x}$ (the component of the force of wind and flow in $\mathrm{x}$ direction), $f_{0 y}$ in $\mathrm{y}$ direction(the component of the force of wind and flow in y direction), $G_{0}-P_{0}$ in z direction. As for the steel drum,the component of $T_{n+2}$ (we suppose there are n section anchor chains)in x direction is $F_{H}+\sum_{m=1}^{i} f_{m x}+f_{0 x}, \sum_{m=1}^{i} f_{m y}+f_{0 y}$ in y direction, $F_{H} \tan \theta_{1}+\sum_{m=1}^{i}\left(G_{m}-P_{m}\right)+G_{0}-P_{0}$ in z direction.

At last,we analysis the stress condition of the buoy,we mark out the force of the buoy in figure7.

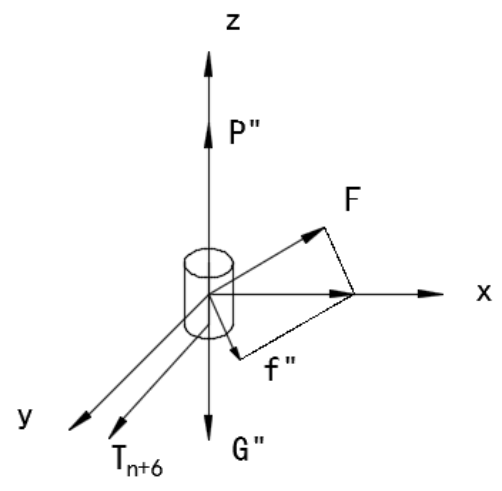

FIGURE7.stress diagram of the buoy

From the figure, we analysis the force in $\mathrm{z}$ direction.we conclude:

$$
F_{H} \tan \theta_{1}+\sum_{m=1}^{i}\left(G_{m}-P_{m}\right)+G_{0}-P_{0}=\rho_{l} g S^{\prime} h
$$

\section{Moment-equilibrium}

For the similar stress condition,the analysis of the moment to the part of the system is similar too. ${ }^{[2]}$ In order to solve the problem,we build a space coordinate system like following:
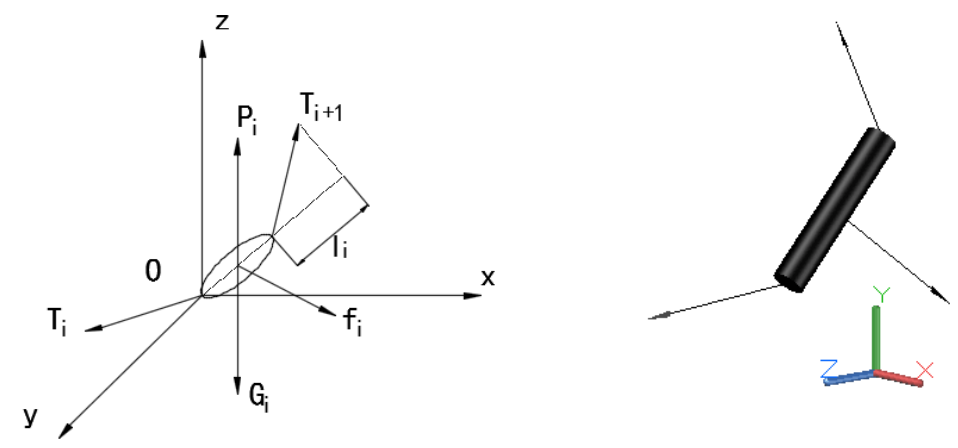

FIGURE8.moment-equilibrium of the anchor chain

We set the top of the anchor chain as coordinate $(x, y, z)$, and $x, y, z$ satisfy the equation $l_{i}^{2}=x^{2}+y^{2}+z^{2}, l_{i}$ is the length of the anchor chain ${ }^{[3]}$.We regard some forces on the centroid of the chain,like wind,flow,buoyancy and its gravity, we view them as a composition $\left(a_{3}, b_{3}, c_{3}\right)$. We know there different tension in its two ends, because of the balance of the there forces to keep the chain,they must in a plane. We use vector to describe the tension on the end of the chain. We move $T_{i+1}$ to the coordinate origin as $T_{i+1}^{\prime}$.We can show them in the following:

$$
\begin{aligned}
& \vec{T}_{i}\left(F_{H}+\sum_{m=1}^{i-1} f_{m x}, \sum_{m=1}^{i-1} f_{m y}, F_{H} \tan \theta_{1}+\sum_{m=1}^{i-1}\left(G_{m}-P_{m}\right)\right) \\
& \vec{T}_{i+1}^{\prime}\left(F_{H}+\sum_{m=1}^{i} f_{m x}, \sum_{m=1}^{i} f_{m y}, F_{H} \tan \theta_{1}+\sum_{m=1}^{i}\left(G_{m}-P_{m}\right)\right)
\end{aligned}
$$

In order to simple the model,we use $\left(a_{1}, b_{1}, c_{1}\right)\left(a_{2}, b_{2}, c_{2}\right)$ to describe $\vec{T}_{i}$ and $\vec{T}_{i+1}^{\prime}$, in this way,we can use moment-equilibrium to get a quadratic equation with three variables. 


$$
\left\{\begin{array}{l}
l_{i}^{2}=x^{2}+y^{2}+z^{2} \\
x_{0} x+y_{0} y+z_{0} z=0 \\
2 l_{i} \sqrt{a_{2}^{2}+b_{2}^{2}+c_{2}^{2}-\left(x a_{2}+y b_{2}+z c_{2}\right)^{2}}=l_{i} \sqrt{a_{3}^{2}+b_{3}^{2}+c_{3}^{2}-\left(x a_{3}+y b_{3}+z c_{3}\right)^{2}}
\end{array}\right.
$$

In this way,we can get the value of z.By analysis all parts of the system,we can conclude $\sum_{m=1}^{n+5} z_{m}+h=H$.

\section{Example calculation}

If the depth of water is at the range of 16 to 20 meter,the maximum of wind is $36 \mathrm{~m} / \mathrm{s}$, the maximum of flow is $1.5 \mathrm{~m} / \mathrm{s}$.In this method, we conclud that there are 258 section chains and the ball's mass is $4630.811 \mathrm{~kg}$.

We test out the system in differt condition like this:

TABLE2.System competition(depth $16 \mathrm{~m}$, wind speed $36 \mathrm{~m} / \mathrm{s}$ )

\begin{tabular}{ccccc}
\hline flow speed(m/s) & drum tilt angle & buoy movement range(m) & section of hanging chain & \multicolumn{2}{c}{$\begin{array}{l}\text { Steel pipe inclination } \\
\text { angle }\end{array}$} \\
\hline 1.5 & 5.0000 & 25.4955 & 258 & 4.67 \\
1.0 & 2.7751 & 24.2628 & 193 & 2.61 \\
0.5 & 1.3692 & 23.1058 & 149 & 1.31 \\
0.0 & 0.9074 & 22.3814 & 131 & 0.90 \\
\hline
\end{tabular}

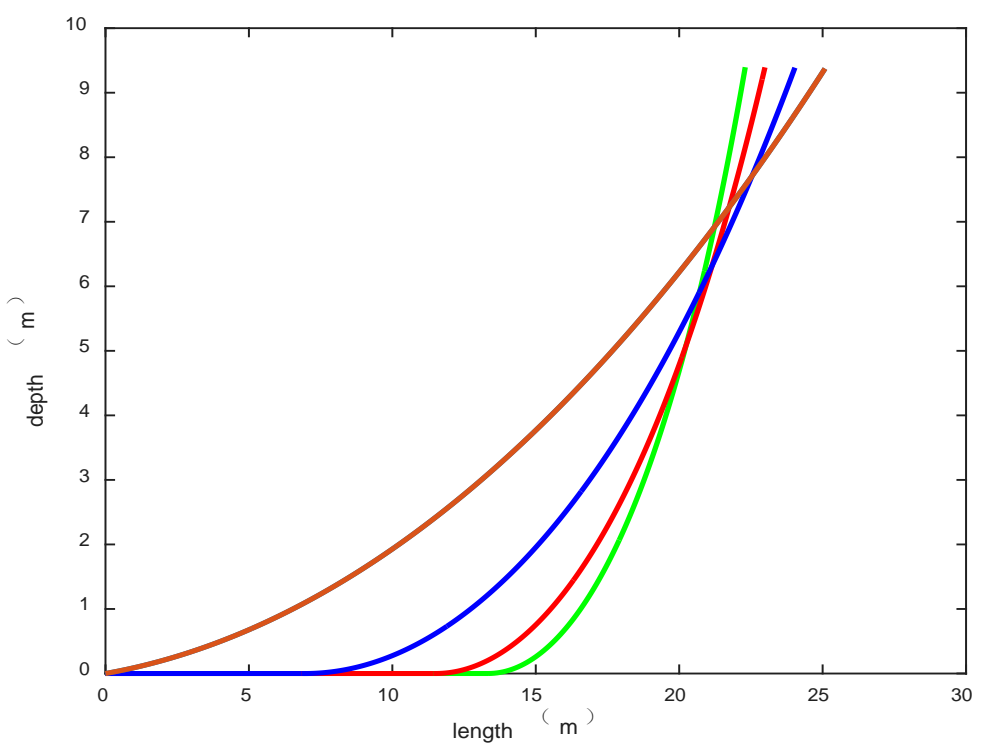

FIGURE9.the shape of the chain in the water(brown $1.5 \mathrm{~m} / \mathrm{s}$,blue $1.0 \mathrm{~m} / \mathrm{s}$, red $0.5 \mathrm{~m} / \mathrm{s}$,green $0.0 \mathrm{~m} / \mathrm{s}$ )

\section{CONCLUSION}

By using the force balance and moment balance, the spatial model of the mooring system is established.We can understand every parts of the system,both in forece and gesture.In this way,we can design mooring system in different enviroment,to keep the instrument work well,and get the different parts' parameter.what's more, we should care that sometimes the total section chain are not hanging,there are some chain in the seabed.

\section{ACKNOWLEDGMENTS}

First and foremost, I would like to show my deepest gratitude to my teacher, Mr.Weiliang Liu, a respectable, responsible and resourceful scholar, who has provided me with valuable guidance in every stage of the writing of this thesis. Without his enlightening instruction, impressive kindness and patience, I could not have completed my thesis. His keen and vigorous academic observation enlightens me not only in this thesis but also in my future study. I would also like to thank all my teachers who have helped me to develop the fundamental and essential academic competence.

\section{REFERENCES}

[1]Detao Luo,Jiading Chen,Static analysis of mooring positioning system,Ship Engineering,Vol 3,1982

[2]Ruijie Zheng,Internal forces analysi of mooring system,Dalian University of Technology,2006(6) 
[3]Triantafyllou MS.Preliminary Design of Mooring System[J].1982,26(1):25-36 